\title{
ANÁLISE EXPERIMENTAL DA INFLUÊNCIA DA TECNOLOGIA HOMEPLUG NA QUALIDADE DA ENERGIA ELÉTRICA
}

\author{
Antonio Luiz Pereira de Siqueira Campos \\ Professor do CEFET-RN. Doutor em Engenharia Elétrica. \\ antonioluiz@cefetrn.br \\ Augusto César Fialho Wanderley \\ Professor do CEFET-RN. Graduado em Engenharia Elétrica. \\ augusto@cefetrn.br \\ Rafael Briani Biondi \\ Tecnólogo em Automação Industrial - CEFET-RN. \\ rafa_alr@yahoo.com.br
}

\begin{abstract}
RESUMO
Trabalhos recentes têm estudado características do meio elétrico para a transmissão de dados, ruídos e técnicas empregadas na comunicação pela rede elétrica. A maioria dos trabalhos limita-se a fazer testes de desempenho do protocolo. Diversos pesquisadores têm se preocupado com a influência das transmissões de dados na rede elétrica sobre as comunicações sem fio, mas ninguém tem se preocupado com a influência dessas transmissões na qualidade da energia elétrica. Este trabalho apresenta resultados preliminares sobre o efeito produzido por redes de computadores que utilizam a tecnologia Homeplug 1.0 na qualidade da energia elétrica. Para isso, foram escolhidos parâmetros apropriados para análise da qualidade da energia elétrica, bem como, foram definidas as formas de se obter esses parâmetros. Foi montada uma rede de computadores, com modem's PLC, no CEFET-RN e realizados testes iniciais para medição da qualidade de energia elétrica. Resultados numéricos são apresentados.
\end{abstract}

PALAVRAS-CHAVE: Qualidade da energia elétrica, Homeplug 1.0, Redes PLC.

\section{EXPERIMENTAL ANALISYS OF HOMEPLUG TECHNOLOGY INFLUENCE ON ELECTRICAL ENERGY QUALITY}

\begin{abstract}
Recent works have studied electrical characteristics of the medium for data transmission, noise and communication techniques employed in powerline communication. Most of those works is limited to do performance testing of the protocol. Several researchers have been concerned about the influence of power line communications on the wireless communications, but no one has been concerned with the influence of such transmissions in the quality of electricity. This paper presents preliminary results on the effect produced by computer networks that use the Homeplug 1.0 technology in the quality of electricity. For this, appropriate parameters were chosen for analysis of the quality of electricity and have been defined ways of achieving these parameters. A computer network was mounted with at the CEFET-RN and conducted initial tests to measure the quality of electricity. Numerical results are presented.
\end{abstract}

KEY-WORDS: Quality of electricity, Homeplug 1.0, PLC Networks. 


\section{ANÁLISE EXPERIMENTAL DA INFLUÊNCIA DA TECNOLOGIA HOMEPLUG NA QUALIDADE DA ENERGIA ELÉTRICA}

\section{INTRODUÇÃO}

Uma demanda crescente por conectividade em ambientes domésticos vem surgindo devido à necessidade de compartilhar recursos e acesso à Internet em banda larga. Assim, o paradigma de redes domiciliares foi criado privilegiando características importantes como a onipresença de pontos de acesso à rede na residência (CAMPISTA, 2004). O grande mercado para os próximos anos em redes domiciliares é a interconexão, em rede e com qualidade de serviço, de equipamentos de áudio e vídeo. O custo é fator primordial em redes domiciliares e, conseqüentemente, a realização de obras civis para instalação de novos cabos pode ser decisiva na escolha da tecnologia a ser adotada.

As redes domiciliares através da rede elétrica (Redes PLC - Power Line Communication) são uma alternativa de acesso que têm a grande vantagem de não precisarem de novos cabos para a comunicação. Redes domiciliares é a interconexão de dispositivos domésticos restritos a uma área residencial, limitada a $300 \mathrm{~m}$. Prevêem-se aplicações de controle e monitoramento de eletrodomésticos, interconexão de computadores, aparelhagem de som e vídeo, telefonia (voz sobre IP) e compartilhamento de acesso a Internet em banda larga. Hoje o mercado está voltado para as aplicações de interconexão de computadores e acesso à Internet, enquanto para um futuro próximo espera-se poder atender a aplicações de vídeo.

A idéia de usar a fiação elétrica como meio de comunicação não é nova. Já em 1838, surgiu o primeiro medidor de consumo remoto. A fiação elétrica é um meio bastante hostil para comunicações de dados, e estas aplicações pioneiras somente foram possíveis por requererem baixas taxas de transmissão. Recentemente, surgiram as primeiras propostas de se utilizar complexas técnicas de modulação e correção de erros para permitir o aumento da taxa de transmissão. Isto possibilitou a interconexão de computadores e o compartilhamento de acesso a Internet usando a fiação elétrica, com isto, surgiu o Padrão HomePlug.

O padrão HomePlug (LEE, 2002) está em sua primeira versão e foi desenvolvido pela HomePlug Powerline Alliance. Este padrão define o método de acesso ao meio e especificações da camada física. Sua principal preocupação é a robustez da transmissão de dados para compensar as adversidades do canal. Segundo Pavlidou o meio elétrico tende a ser pior que o meio sem fio em termos de atenuação e ruído (PAVLIDOU, 2003).

Os principais fatores que dificultam a propagação de sinal são: a atenuação, o ruído e a distorção. Para resolver estes problemas o padrão HomePlug usa técnicas avançadas de modulação como o OFDM (Orthogonal Frequency Division Multiplexing) e controle de acesso ao meio por CSMA/CA (Carrier Sense Multiple Access with Collision Avoidance). $\mathrm{O}$ controle de acesso ao meio por CSMA/CA é geralmente utilizado, pois devido à atenuação, não é possível garantir a detecção de colisão (BINGHAM, 1990).

Este trabalho visa promover experimentos em um ambiente fechado, com a presença de transmissão PLC e sem a presença desta transmissão, para que se seja possível uma comparação de resultados, chegando à conclusão sobre os graus de interferência causados na rede elétrica e se estas interferências são capazes de prejudicar ou até mesmo interferir 
em um processo residencial ou produtivo. Os fatores analisados são a Taxa de Distorção Harmônica (THD) e um possível aumento na presença de freqüências harmônicas.

\section{PADRÃO HOMEPLUG 1.0}

A tecnologia PLC começou a ser utilizada na década de 1920 para transmissão de voz (modulação AM) em redes de alta tensão. Mais tarde começou a ser utilizada em aplicações de telemetria e controle. Apenas na década de 1990 foi criado o primeiro protótipo de um modem que trabalhava com uma taxa modesta de 60 bps e transmitia a uma distância máxima de $1 \mathrm{~km}$. Dentre as principais melhorias do protótipo estão a capacidade de transmissão bidirecional, a utilização de freqüências mais elevadas e menores níveis de potência do sinal transmitido (LIN, 2003).

Até pouco tempo atrás não havia nenhum padrão para essa tecnologia, o que significa que cada empresa desenvolvia sua própria tecnologia PLC. A iniciativa de criar uma tecnologia unificada teve início com a criação do grupo HomePlug Alliance, formado por algumas das maiores empresas de tecnologia do mundo como LG, Motorola, Linksys, Intel, Sansung, Sharp, Texas Instruments, etc.

Desta união foi criado o padrão HomePlug 1.0, para distribuição de internet residencial, HomePlug AV destinado para transmissões de dados, áudio e vídeo (HDTV) “in-home” e HomePlug BPL, que é responsável pelo acesso à internet diretamente pela concessionária de energia. Neste trabalho, será apresentado o padrão Homepug 1.0.

O padrão Homeplug 1.0 foi o primeiro padrão PLC desenvolvido pela HomePlug Alliance, um consórcio criado em 2000 composto de empresas de grande participação no mercado internacional de tecnologia como Linksys, Intel, LG, Sansung, Motorola, etc com o objetivo de criar um padrão para a tecnologia PLC. Esse protocolo foi concebido para distribuir internet em um ambiente residencial. Na verdade ele veio para tentar suprir um "vácuo" entre as tecnologias "com fio" e "sem fio".

No desenvolvimento de parte de uma rede em que não é possível a passagem de cabos comuns e o sinal Wireless não é captado com qualidade, dispositivos HomePlug 1.0 oferecem uma solução de qualidade. Utilizando a fiação elétrica já existente no local (sem novos fios) esse padrão é capaz de alcançar uma taxa nominal de 13,78 Mbps na camada física.

Para conseguir essas taxas o padrão utiliza multiplexação OFDM com as modulações derivadas do PSK (Phase Shift Keying): DBPSK (Differential Binary Phase Shift Keying) e DQPSK (Differential Quadrature Phase Shift Keying). Enquanto que a modulação DBPSK consegue transmitir 1 bit por símbolo por portadora, o DQPSK consegue modular 2 bits por símbolo por portadora.

O acesso ao meio é feito através de uma versão aprimorada do CSMA/CA, na qual um registrador chamado DC (Deferal Counter) é implementado para impedir que pacotes de menor prioridade fiquem esperando serem transmitidos por muito tempo.

Para detecção e correção de erros o protocolo HomePlug 1.0 utiliza como FEC (Forward Error Correction) o Código Convolucional concatenado com Reed-Solomon que, embora consiga garantir qualidade no recebimento da informação, reduz a taxa efetiva de dados. 
$\mathrm{Na}$ camada física o Homeplug 1.0 utiliza 128 subportadoras igualmente espaçadas ocupando a faixa de 0 a $25 \mathrm{MHz}$. Destas, apenas 84 são escolhidas depois de uma análise periódica do meio. Além disso, 8 subportadoras não são utilizadas devido à interferência de algumas delas nas transmissões de rádio amador, restando ao final 76 subportadoras. $\mathrm{Na}$ camada MAC o protocolo utiliza 2 bits para implementar 4 níveis de prioridade. Assim, cada nível de prioridade do padrão HomePlug 1.0 encapsula dois níveis de prioridade do padrão 802.1Q (VLAN).

Em uma situação em que o meio apresente grandes dificuldades para transmitir o sinal, o equipamento entra em um modo de operação especial chamado ROBO (ROBust OFDM). Em modo ROBO o sinal passa a ser transmitido com maior redundância, o que causa uma queda significativa na taxa de transmissão. Para garantir essa robustez o protocolo passa a modular o sinal apenas em DBPSK, que apresenta menor sensibilidade ao ruído do que o DQPSK. Além disso, cada bit passa a ser repetido 4 vezes. Somando-se a isso a taxa de codificação passa a ser de $50 \%$, isto quer dizer, os dados de redundância serão responsáveis por metade dos dados enviados. Todas essas alterações no protocolo, para garantir o recebimento dos dados, reduzem a taxa de transmissão para, aproximadamente, 0,9 Mbps.

Outro ponto a favor do HomePlug 1.0 é o fato de não interferir com o sinal de outros protocolos de transmissão PLC como o X-10 e LonWorks.

Como foi dito anteriormente, segundo o padrão HomePlug 1.0, a taxa de transmissão na camada física é de 13,78 Mbps. A Tabela 1 mostra a taxa de transmissão nas principais camadas:

Tabela 1: Taxas de Transmissão Obtidas com o Homeplug 1.0.

\begin{tabular}{|c|c|}
\hline CAMADA & VAZÃO (Mbps) \\
\hline Física & 13,78 \\
\hline Enlace & 8,2 \\
\hline Transporte & 6,2 \\
\hline
\end{tabular}

Outro ponto importante sobre esse protocolo diz respeito ao tipo de criptografia utilizado. A criptografia utilizada no HomePlug 1.0 é a DES (Data Encryption Standard) de 56 bits. Essa criptografia foi criada pela IBM e implantada pelos EUA, em 1977, para criptografia padrão de informações não-confidenciais e é considerada, por muitos autores, como sendo uma criptografia não segura.

\section{PARÂMETROS DE QUALIDADE DE ENERGIA ELÉTRICA}

A definição de qualidade da energia elétrica pode ser vista de diferentes aspectos. A concessionária de energia elétrica se preocupa com a confiabilidade do sistema elétrico. Para o consumidor residencial a definição diz respeito à continuidade no fornecimento de energia elétrica. Para os processos industriais a falta de energia elétrica ou mesmo a oscilação nos parâmetros elétricos podem ser suficientes para causar grandes prejuízos financeiros.

Portanto, qualidade da energia elétrica é definida como qualquer problema na energia manifestado por desvios na tensão, corrente ou freqüência, que resultam na falha ou operação inadequada de equipamentos do usuário (DUGAN, 1996). 
A qualidade da energia elétrica aborda fenômenos eletromagnéticos que são divididos nas seguintes categorias: transitórios, variações de curta-duração e de longa-duração, desbalanceamento de tensão, distorções na forma de onda, flutuações de tensão e variações na freqüência.

Estas categorias por sua vez abrangem várias espécies de fenômenos que classificam e descrevem claramente os distúrbios eletromagnéticos que causam problemas de qualidade da energia elétrica.

Os fenômenos comumente encontrados nas instalações elétricas referentes à qualidade da energia elétrica são apresentados na Tabela 2, juntamente com suas características (ALDABÓ, 2001):

Tabela 2: Características dos Fenômenos de Qualidade de Energia Elétrica.

\begin{tabular}{|l|l|l|}
\hline \multicolumn{1}{|c|}{ FENÔMENO } & \multicolumn{1}{|c|}{ DURAÇÃO TÍPICA } & \multicolumn{1}{|c|}{ AMPLITUDE DA TENSÃO } \\
\hline Harmônicas & Regime & $0-20 \%$ \\
\hline Interharmônicas & Regime & $0-2 \%$ \\
\hline $\begin{array}{l}\text { Afundamento de } \\
\text { tensão (sag) }\end{array}$ & 0,5 ciclo - 1 minuto & $0,1-0,9 \mathrm{pu}$ \\
\hline $\begin{array}{l}\text { Elevação de } \\
\text { tensão (swell) }\end{array}$ & 0,5 ciclo - 1 minuto & $1,1-1,8 \mathrm{pu}$ \\
\hline Subtensão & Maior do que 1 minuto & $0,8-0,9 \mathrm{pu}$ \\
\hline Sobretensão & Maior do que 1 minuto & $1,1-1,2 \mathrm{pu}$ \\
\hline Interrupção de & 0,5 ciclo - 1 minuto & Menor do que $0,1 \mathrm{pu}$ \\
\hline $\begin{array}{l}\text { Flutuações de } \\
\text { tensão }\end{array}$ & Intermitente & $0,1-7 \%$ \\
\hline
\end{tabular}

O monitoramento da qualidade da energia elétrica deve ser realizado com o objetivo de caracterizar as variações na rede elétrica. Este monitoramento deve atender as seguintes atividades:

- Conhecimento das instalações elétricas;

- Escolha do(s) local(is) de instalação dos equipamentos de medição;

- Duração e quantidade das medições;

- Análise dos resultados das medições e

- Aplicação de soluções corretivas.

O conhecimento das instalações elétricas abrange o estudo dos diagramas unifilares e trifilares e a inspeção dos vários sistemas e tipos de cargas elétricas. A elaboração de um histórico dos distúrbios observados ajuda no planejamento das ações. O(s) local(is) de instalação dos equipamentos de medição permite determinar a abrangência do(s) problema(s), ou seja, até onde os seus efeitos são detectados. O primeiro local deve ser o quadro geral. Se apenas um equipamento é atingido seu circuito de alimentação deve ser analisado. O tempo de monitoração também é um fator importante, pois a duração dos fenômenos é bastante diversificada. Aldabó afirma que para observar um problema devemos monitorar a tensão; para determinar a causa devemos monitorar a tensão e a corrente (IEEE, 1992). 
Devido a grande variedade de distúrbios relacionados à qualidade da energia elétrica, também é grande a quantidade de soluções adotadas para a sua correção. Dentre estas soluções podemos citar: equilíbrio das cargas elétricas, instalação de reguladores de tensão, supressores de surto, filtros, sistema de fornecimento ininterrupto de energia (UPS) etc. (IEC, 2003).

\section{METODOLOGIA DOS TESTES}

Com o objetivo de analisar os efeitos produzidos por uma rede de computadores utilizando tecnologia PLC, na qualidade da energia elétrica do CEFET-RN, a metodologia adotada prevê várias medições dos parâmetros elétricos envolvidos. Estas medições foram realizadas na presença e na ausência de transmissões PLC e o local escolhido para as medições foi o Quadro de Distribuição Geral (QDG) do laboratório de eletromecânica.

O equipamento que foi utilizado para realizar as medições foi um analisador de energia elétrica, modelo 808, distribuído pela HOMIS. Este equipamento, além de medir as grandezas elétricas usuais (tensão, corrente, potência e energia), permite a medição de harmônicas, afundamento e elevação de tensão e transitórios. Através de um software fornecido com o equipamento podem-se visualizar em um computador todas as grandezas medidas pelo instrumento.

O diagrama da Figura 1 ilustra como foram realizadas as medições no QTL, no circuito que alimenta os equipamentos.

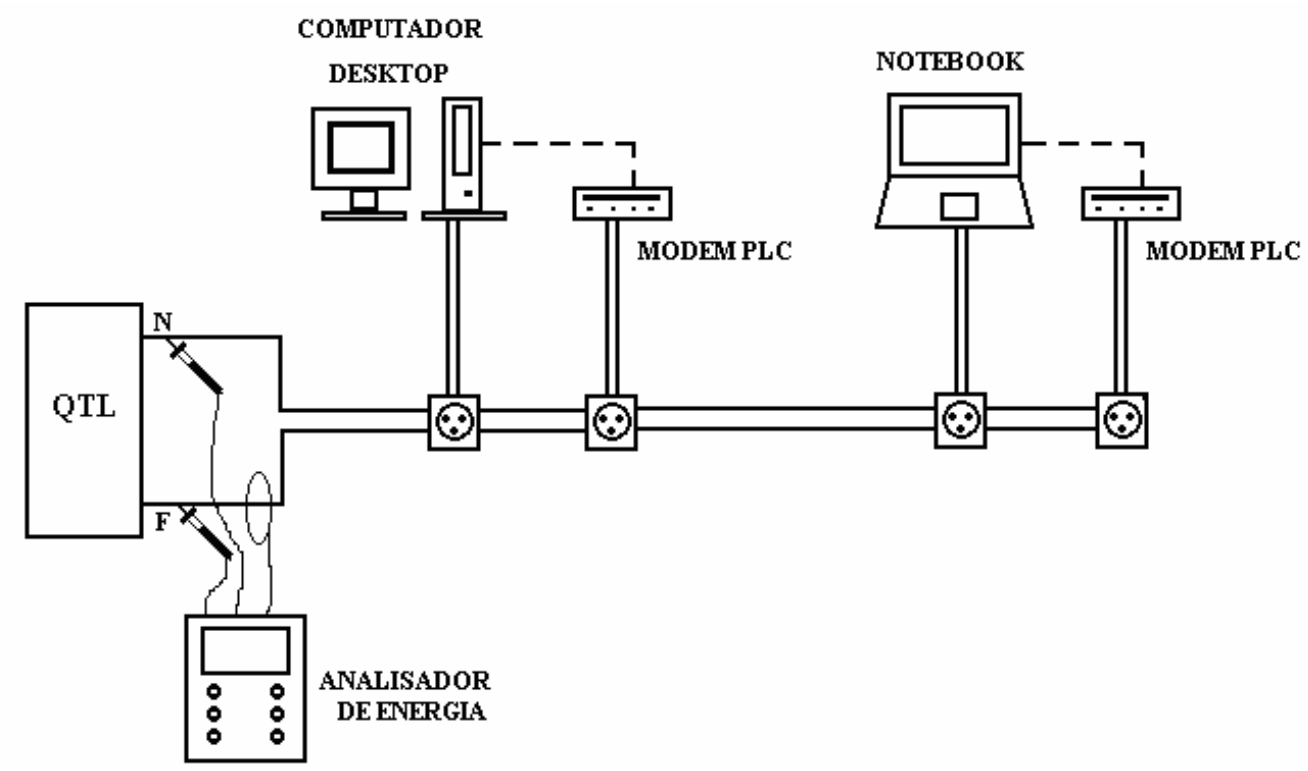

Figura 1 - Diagrama para medição no QTL.

Medições foram realizadas no QTL, visando detectar possíveis distúrbios causados apenas por um computador e também quando os dois computadores estiverem ligados. Após estas medições, os modem's foram conectados aos seus computadores e foram iniciadas transmissões de arquivos entre eles para que novas medições pudessem ser feitas. Em todas as medições os dados foram armazenados no analisador de energia e somente depois analisados com o auxílio do software. A Figura 2 mostra o diagrama de ligação do analisador de energia em quadros trifásicos. 


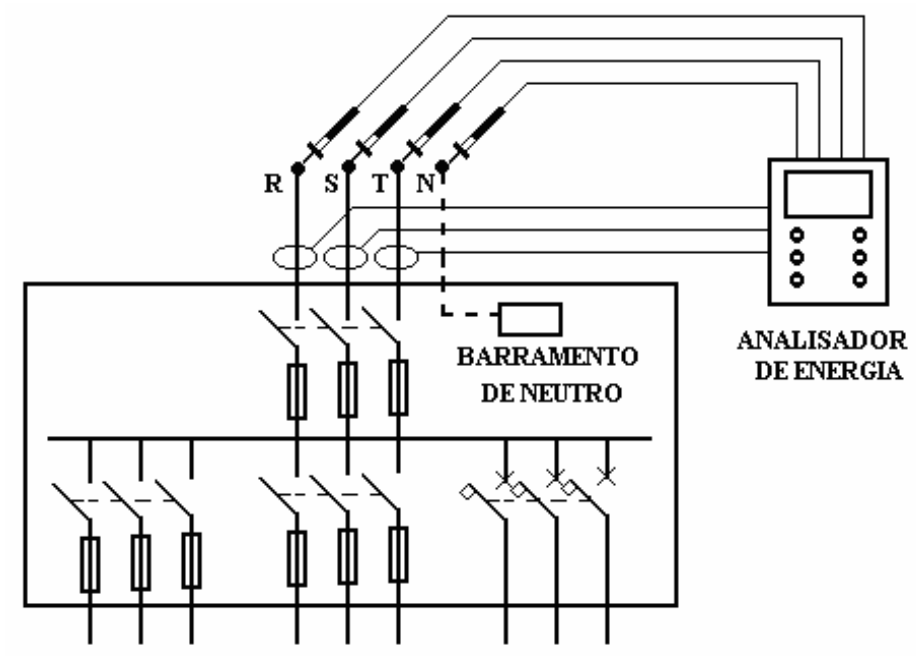

Figura 2 - Conexão do analisador de energia em quadro trifásico.

\section{ANÁLISE DOS RESULTADOS}

Nesta seção são apresentados os resultados obtidos a partir das medições efetuadas no sistema elétrico do Laboratório de Eletromecânica do CEFET / RN. Para esta análise foi escolhida a técnica das medianas, que consiste em se obter o valor central dos dados para representar toda a classe dos mesmos, provocando uma maior concordância dos resultados.

Primeiramente, foram analisados os diagramas de espectros harmônicos presentes na tensão e na corrente das três fases do sistema elétrico e posteriormente as taxas de distorção harmônica total (THD) das mesmas fases, para que se pudesse chegar a alguma conclusão.

Para a primeira fase, os diagramas de espectro da tensão e da corrente são mostrados nas figuras 3 e 4, respectivamente. As figuras ilustram a THD antes e durante as transmissões PLC. Nesses diagramas pode-se observar que as harmônicas presentes, tanto na tensão, como na corrente da primeira fase, não sofreram variações consideráveis a ponto de interferir ou até mesmo desestabilizar o sistema elétrico, tendo em vista que as mesmas sofreram variações menores que um ponto percentual.

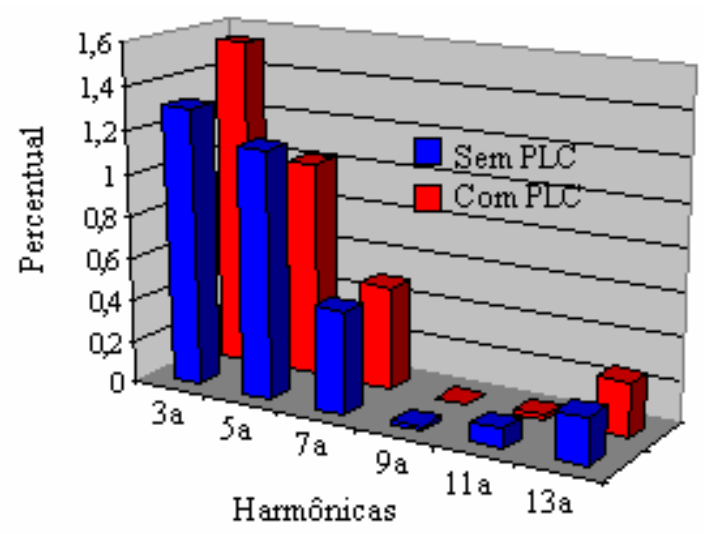

Figura 3 - Diagrama espectral das harmônicas presentes na tensão da primeira fase até a $13^{a}$ harmônica. 


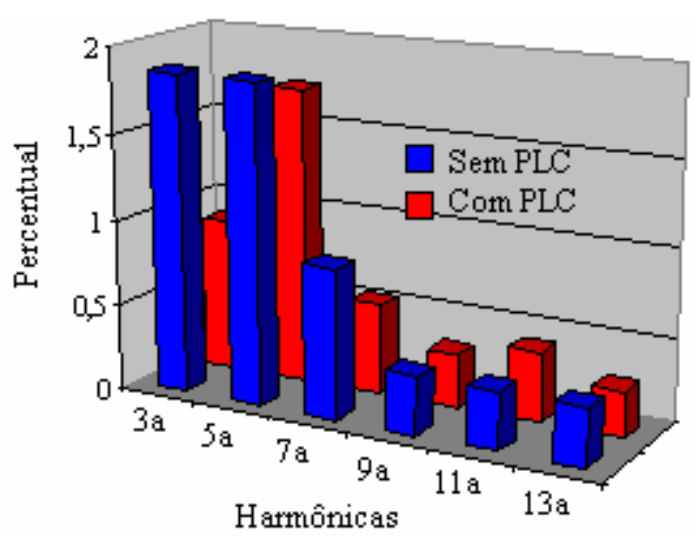

Figura 4 - Diagrama espectral das harmônicas presentes na corrente da primeira fase até a $13^{\mathrm{a}}$ harmônica.

Na Figura 5 pode-se observar que a $3^{\mathrm{a}}, 5^{\mathrm{a}}$ e $13^{\mathrm{a}}$ harmônicas sofreram um aumento durante as transmissões PLC, enquanto que as demais permaneceram ligeiramente menores. Já na Figura 6, pode-se ver que todas as harmônicas sofreram um aumento durante as transmissões PLC. Além disso, pode-se notar uma maior presença de harmônicas na corrente.

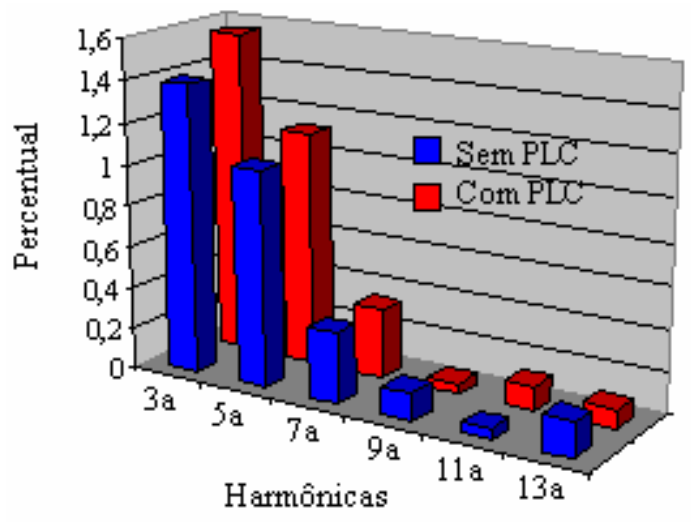

Figura 5 - Diagrama espectral das harmônicas presentes na tensão da segunda fase até a $13^{\mathrm{a}}$ harmônica. 


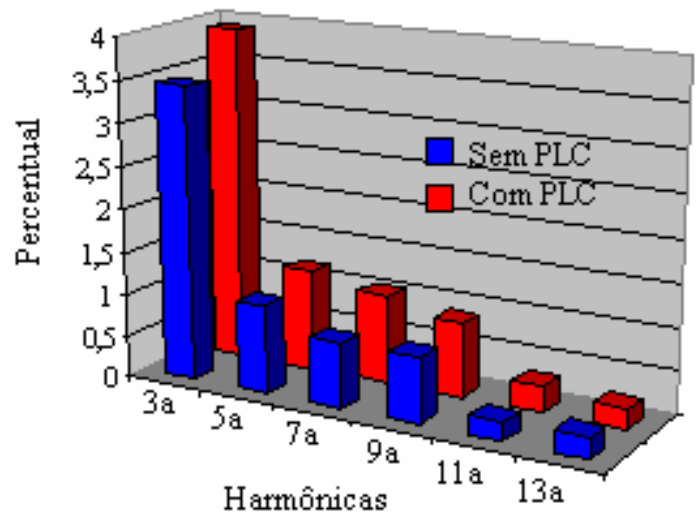

Figura 6 - Diagrama espectral das harmônicas presentes na corrente da segunda fase até a $13^{\mathrm{a}}$ harmônica.

Para a terceira fase, os diagramas de espectro da tensão e da corrente são mostrados nas figuras 7 e 8 , respectivamente.

Na Figura 7 pode-se observar que a todas as harmônicas, com exceção da 9a , sofreram um aumento durantes as transmissões PLC. Já na Figura 8, pode-se ver que todas as harmônicas sofreram uma redução durante as transmissões PLC. Além disso, pode-se notar uma maior presença de harmônicas na corrente, sendo que todas ultrapassaram 1\%.

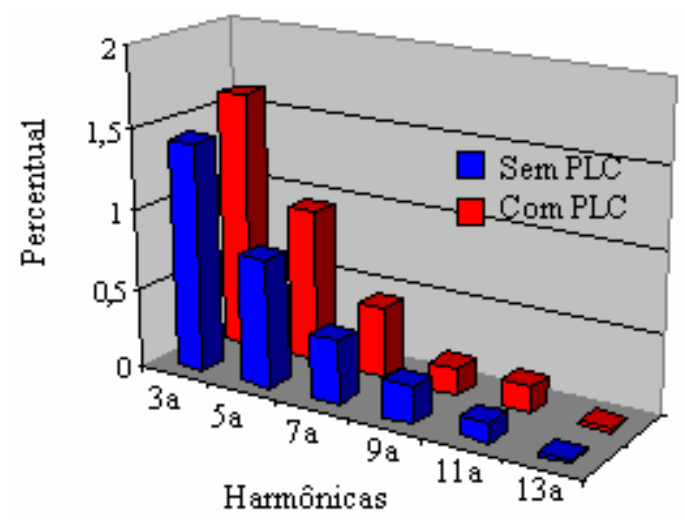

Figura 7 - Diagrama espectral das harmônicas presentes na tensão da terceira fase até a $13^{\mathrm{a}}$ harmônica. 


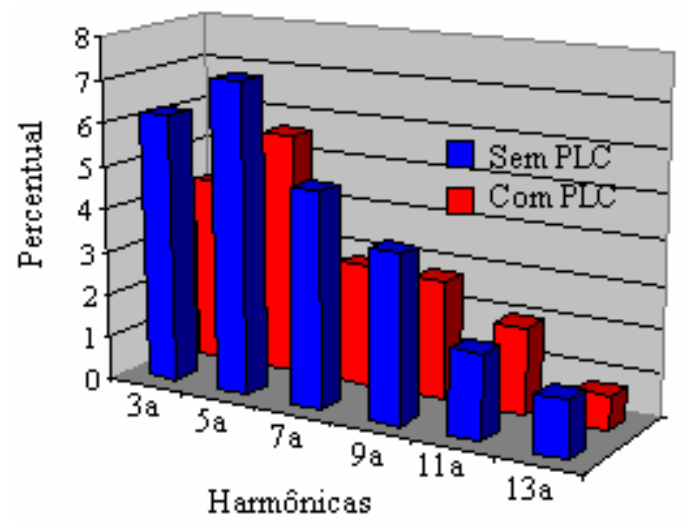

Figura 8 - Diagrama espectral das harmônicas presentes na corrente da terceira fase até a $13^{\mathrm{a}}$ harmônica.

Por fim, na Tabela 3 tem-se a taxa de distorção harmônica total para as tensões e correntes de cada fase. Pode-se observar que as tensões sofreram um ligeiro aumento na THD, enquanto que as correntes não, para medições com transmissões PLC.

Tabela 3: Características dos Fenômenos de Qualidade de Energia Elétrica.

\begin{tabular}{|l|c|c|}
\hline \multirow{2}{*}{\multicolumn{1}{|c|}{ GRANDEZA }} & \multicolumn{2}{|c|}{ PARÂMETRO } \\
\cline { 2 - 3 } & THD sem Transmissão & THD com transmissão \\
\hline Tensão da Fase 1 & $1,9 \%$ & $2,1 \%$ \\
\hline Corrente da Fase 1 & $3,0 \%$ & $3,0 \%$ \\
\hline Tensão da Fase 2 & $1,9 \%$ & $2,1 \%$ \\
\hline Corrente da Fase 2 & $6,7 \%$ & $5,8 \%$ \\
\hline Tensão da Fase 3 & $1,9 \%$ & $2,1 \%$ \\
\hline Corrente da Fase 3 & $13,6 \%$ & $8,9 \%$ \\
\hline
\end{tabular}

\section{CONCLUSÕES}

Após a análise dos dados pode-se perceber que houve um pequeno aumento significativo na Taxa de Distorção Harmônica Total (THD) durante as transmissões. Algumas freqüências harmônicas dos sistemas elétricos nos quais as redes estão transmitindo também sofreram um pequeno aumento. Entretanto, um número maior de medições mostra-se necessário para se ter uma conclusão mais significativa sobre o problema, tendo em vista que só foram analisados fatores de aumento na presença de harmônicas e na THD do sistema elétrico, deixando-se de lado outros fatores de qualidade de energia. Este trabalho é um início para esta linha de pesquisa e seus resultados podem ser de úteis para pesquisadores que posteriormente decidam estudar as redes PLC e sua co-relação com os fatores de qualidade de energia. Como continuidade deste trabalho sugere-se uma radiografia do local analisado no que diz respeito à qualidade de energia elétrica. Além disso, a medição de outros parâmetros de qualidade de energia elétrica devem ser monitorados. 


\section{REFERÊNCIAS BIBLIOGRÁFICAS}

1. ALDABÓ, R. Qualidade na energia elétrica. São Paulo: Artliber, 2001.

2. BINGHAM, J. A. C. Multicarrier modulation for data transmission: an idea whose time has come. IEEE Communications Magazine, v.28, n.5, pp. 5 - 14, 1990.

3. CAMPISTA, M. E. M. Uma Análise da Capacidade de Transmissão na Rede de Energia Elétrica Domiciliar. In: XXI SIMPÓSIO BRASILEIRO DE TELECOMUNICAÇÕES - SBrT 2004, 2004, Belém.

4. DUGAN, R. C. MCGRANAGHAN, M. F. e BEATY, H. W. Electrical power systems quality. New York: McGraw-Hill, 1996.

5. INSTITUTE OF ELECTRICAL AND ELECTRONICS ENGINEERS. IEEE Std. 519: IEEE recommended practices and requirements for harmonic control in electrical power systems. New York, 1992.

6. INTERNATIONAL ELECTROTECHNICAL COMMISSION. IEC 61004-4-30: Electromagnetic compatibility (EMC) - Part 4-30: Testing and measurement techniques

- Power quality measurement methods. 2003.

7. LEE, M. K. et. al. HomePlug 1.0 Powerline Communication LANs -Protocol Description and Performance Results version 5.4. International Journal of Communication Systems, 2002, pp. 1 - 25.

8. LIN, Y. J. LATCHMAN, H. A. e NEWMAN, R. E. A Comparative Performance Study of Wireless and Power Line Networks. IEEE Communications Magazine, v.41, n.4, pp. $54-63,2003$.

9. PAVLIDOU, N. et. al. Power Line Communications: State of the Art and Future Trends. IEEE Communications Magazine, pp. 34 - 40, 2003. 